\title{
Wearing a KN95/FFP2 Facemask Has Subtle Effects on Functional Salience Network Interaction
}

Sven HALLER ( $\square$ sven.haller@me.com )

CIMC - Centre d'Imagerie Médicale de Cornavin

Marie-Louise MONTANDON

University of Geneva

Cristelle RODRIGUEZ

University of Geneva

Panteleimon GIANNAKOPOULOS

University of Geneva

\section{Research Article}

Keywords: COVID-19, DMN, FFP2/KN95 facemask, phenomenon, SN, CONN

Posted Date: January 3rd, 2022

DOI: https://doi.org/10.21203/rs.3.rs-1171603/v1

License: (c) (1) This work is licensed under a Creative Commons Attribution 4.0 International License. Read Full License

Version of Record: A version of this preprint was published at European Radiology Experimental on October 10th, 2022. See the published version at https://doi.org/10.1186/s41747-022-00301-0. 


\section{Abstract}

\section{Background}

Wide use of wearing a facemask is one of the many consequences of the COVID-19 pandemic. We used resting-state fMRI to search for subtle changes in brain functional connectivity, expected notably related to the high-level salience (SN) and default mode networks (DMN).

\section{Materials and Methods}

Prospective crossover design resting fMRI study with/without wearing a tight FFP2/KN95 facemask, including 24 consecutive male participants (mean age $\pm S D=29.9 \pm 6.9$ years) at $3 T$. As physiological parameters, respiration frequency and heart rate were monitored. Data analysis was performed using the CONN toolbox.

\section{Results}

Wearing an FFP2/KN95 facemask did not impact respiration or heart rate but resulted in a significant reduction in functional connectivity between the $\mathrm{SN}$ as the seed region and the left middle frontal and precentral gyrus. No difference was found when the DMN, sensorimotor, visual, dorsal attention or language networks were used as seed regions.

\section{Conclusions}

The wearing of tight FFP2/KN95 facemasks in men decreases the functional connectivity of the SN, which is known to be involved in communication, social behavior and self-awareness. This phenomenon was confined to the left frontal and precentral gyrus. Our results imply that the wearing of a facemask could impact our ability to detect and react appropriately to salient stimuli by altering the functional connectivity of the SN.

\section{Introduction}

Wearing a facemask in professional settings was usually restricted to medical personnel, and surgical facemasks were typically used. The COVID-19 pandemic changed this reality, including the wearing of facemasks in daily life interactions and in MRI scanning facilities. Wearing a facemask on a daily basis is not supposed to interfere with cognitive abilities. Whether this practice impacts brain function remains unclear. To date, only one study in eight subjects indicated that it might alter BOLD baseline signals without affective task-related activation ${ }^{1}$. We used resting-state functional magnetic resonance imaging ( $\mathrm{fMRI}$ ) to assess subtle modifications in functional connectivity using a crossover design, i.e., Half of the participants first had no mask followed by mask, and half of the participants had the inverse order.

Since the behavioral consequences of wearing a facemask are minimal, we expected only very subtle changes in brain functional connectivity that should concern high-level brain networks rather than basic 
networks such as visual or motor networks. Consequently, we focused our analysis on the salience network (SN), which is involved in communication, social behavior, and self-awareness through the integration of sensory, emotional and cognitive information ${ }^{2}$, and the default mode network, known for being active when a person is not focused on the outside world. To avoid potential bias of the resting fMRI results due to basic physiologic parameters, we monitored breathing and heart rate during the fMRI runs.

\section{Materials And Methods}

\section{Participants}

This prospective study was approved by the Ethics Committee of the University Hospitals and University of Geneva, Switzerland, the study was in accordance with the Declaration of Helsinki, and all participants gave written informed consent. The study included 24 community-dwelling cases recruited via advertisements in local media. One case was excluded from further analysis due to the incidental finding of an enlarged ventricular system. The final sample included 23 cases (mean age $\pm S D=29.9 \pm 6.9$ years).

\section{Image acquisition}

MR images were acquired using a 3T MRI scanner (MAGNETOM PRISMA, Siemens) at Campus Biotech Geneva (https://www.campusbiotech.ch/). Functional echo-planar imaging had the following essential parameters: 66 slices, slice thickness $=2.0 \mathrm{~mm}$, voxel size $=2.0 \mathrm{~mm} \times 2.0 \mathrm{~mm} \times 2.0 \mathrm{~mm}$, repetition time $=$ $1000 \mathrm{~ms}$, echo time $=32 \mathrm{~ms}$, flip angle $=50^{\circ}$, and field of view $=224 \mathrm{~mm}$, resulting in $7.11 \mathrm{~min}$ per resting-state fMRI run. Each participant performed 2 runs in a crossover design, once with and once without an FFP2/KN 95 facemask. An additionally acquired 3DT1 sequence (208 slices; slice thickness = $1.0 \mathrm{~mm}$; voxel size $=1 \times 1 \times 1 \mathrm{~mm}^{3}$; repetition time $=2300 \mathrm{~ms}$; echo time $=2.26 \mathrm{~ms}$; flip angle $=8^{\circ}$; field of view $=256 \mathrm{~mm}$ ) was used for spatial normalization and registration.

Heart rate and respiration data were collected continuously and simultaneously for resting-state functional imaging using BIOPAC systems (https://www.biopac.com/research/).

\section{Image analysis}

Image analysis was performed using the CONN toolbox version 20b (www.nitrc.org/projects/conn, RRID:SCR_009550).

Data analysis was performed using the standard processing steps as described previously ${ }^{3}$. The data processing included motion correction, spatial filtering, denoising and transformation into NMI standard space using the individual 3DT1 brain sequence for anatomic spatial registration. Then, the functional time series were analyzed using the following steps: 
Fist, we performed a ROI-to-ROI analysis. The ROI-to-ROI connectivity metrics characterize the connectivity between all pairs of ROls among a predefined set of regions. The ROls were defined using the functional connectivity atlas included in the CONN toolbox. This atlas notably includes predefined resting state networks of the SN, DMN, visual and language networks. This ROI-to-ROI analysis examines the functional connection between each pair of ROIs, for example DMN - SN. This pairwise ROI-to-ROI connectivity analysis was compared for FACEMASK versus no FACEMASK.

Second, we performed a seed-to-voxel analysis. This seed-based connectivity analysis aims to investigate functional properties from preselected seed regions. As seed regions, the same functional atlas-based regions were used as described above ( $S N$, DMN, visual and language networks). Similar to the ROI-to-ROI analysis described above, a pairwise comparison for FACEMASK versus no FACEMASK was performed.

Both analyses (ROI-to-ROI and seed-to-voxel) therefore used the same predefined atlas regions as $\mathrm{ROI} / \mathrm{seed}$. The difference between them is that the ROI-to-ROI only investigates functional connectivity between predefined ROIs but cannot detect functional connectivity changes in other (nonpredefined) regions. In contrast, seed-to-voxel analysis is able to detect functional connectivity changes of the preselected seed ROI to any unselected voxel of the brain without reference to predefined functional anatomic regions. Multiple-comparisons correction was applied using the false discovery rate (FDR) ${ }^{4}$.

\section{Results}

There were no differences in heart rate or respiration rate for FACEMASK versus NOMASK.

As a first step, we performed an ROI-to-ROI analysis using predefined atlas-based resting state networks, including the SN and DMN, as well as sensorimotor, visual and language networks. We found no significant direct effect of wearing a facemask within the established resting state networks, including the SN, DMN, sensorimotor, visual, dorsal attention or language networks.

As a second step, we performed a seed-to-voxel analysis using the networks mentioned above as seed regions. The resting $\mathrm{fMRI}$ seed-to-voxel analysis using the $\mathrm{SN}$ as the seed region revealed that FACEMASK versus NOMASK resulted in a significant reduction in a cluster in the left middle frontal and precentral gyrus ( $p<0.0002$ FDR corrected), see figure Figure 1. There were no significant group differences using the DMN or sensorimotor, visual, dorsal attention or language networks as seed regions.

\section{Discussion}

The effect of wearing a tight FFP2/KN95 facemask on brain functional connectivity was significant using the SN but not the DMN, sensorimotor, visual, dorsal attention or language networks as seed regions. Importantly, there were no potentially confounding physiological effects since no group differences were found in heart rate or respiration. 
The only previous study available in this field ${ }^{1}$ specifically assessed the effect of wearing a facemask on functional MRI but with a different approach and objective. This study assessed task-related fMRI during a basic sensory-motor task designed to activate visual, auditory, and sensorimotor cortices in 8 participants. This study did not report significant effects of wearing a facemask on task-related fMRI activation in basic sensorimotor areas. We expected only very subtle changes related to wearing a facemask and those changes to occur in higher-level cognitive centers. The current resting-state fMRI study in 23 cases focusing on higher-level resting networks found significant effects only related to the high-level SN. Of note, and consistent with a previous study ${ }^{1}$, our analysis did not reveal significant differences using the lower-level sensorimotor or visual networks as seed regions.

We expected only very subtle changes related to wearing a facemask, and those changes to occur were related to higher-level cognitive centers. In agreement with our a priori hypothesis, the effect of facemask wearing was confined to an interaction between the $\mathrm{SN}$ as the seed region and the left middle frontal and precentral gyrus. The $\mathrm{SN}$ is thought to facilitate the detection of important environmental stimuli 2,5 . It is mainly involved in detecting and filtering multimodal salient stimuli and mediating the transition from the DMN to the central executive network. Our results might imply that the wearing of a facemask might impact our ability to detect and react appropriately to salient stimuli by altering the functional connectivity of the SN. However, this phenomenon concerned only the connections to the left frontal and precentral gyrus. The significance of this finding remains unclear. Altered functional connectivity of the $\mathrm{SN}$ with frontal and precentral areas was reported as part of a more global cortical disconnection in a variety of neuropsychiatric disorders, including psychosis, poststroke depression and attention deficit syndrome ${ }^{6-8}$. In young controls, the connectivity between the $\mathrm{SN}$ and left frontal as well as precentral areas is thought to be crucial not only for episodic memory skills ${ }^{9}$ but also for working memory activation ${ }^{10}$. Whether changes in restricted parts of $\mathrm{SN}$ functional connectivity may predispose individuals to decreased activation of these cognitive functions in highly demanding situations is still unknown.

Two limitations should be considered when interpreting the present findings. To avoid the well documented gender-related differences in functional connectivity ${ }^{11}$, we included only male participants in this study. Although this procedure makes sense given the small series of cases that accepted this constraining experimental design, it naturally limits the generalizability of the present findings. Moreover, we deliberately used a tight FFP2/KN95 facemask, which many people prefer in the context of the COVID19 pandemic and are requested by most airline companies. We would expect smaller effects on brain functional connectivity when using less tight surgical facemasks, which was the standard facemask in the field of medicine before the COVID-19 pandemic. Future studies with various types of masks in mixed samples and using ad hoc activation paradigms will make it possible to complete these at rest observations, gaining better insight into the impact of facemask wearing on brain activation patterns.

\section{Declarations}




\section{Funding}

This research was carried out with the help of an unrestricted grant from Association Suisse pour la Recherche sur Alzheimer, an unrestricted grant from the Schmidheiny foundation and the Swiss National Foundation grant (Grant No. 320030-169390, and 320030_169876).

\section{References}

1. Law, C. S. W., Lan, P. S. \& Glover, G. H. Effect of wearing a face mask on fMRI BOLD contrast. Neuroimage 229, 117752 (2021).

2. Menon, V. \& Uddin, L. Q. Saliency, switching, attention and control: a network model of insula function. Brain Struct Funct 214, 655-667 (2010).

3. Whitfield-Gabrieli, S. \& Nieto-Castanon, A. Conn: a functional connectivity toolbox for correlated and anticorrelated brain networks. Brain connectivity 2, 125-141 (2012).

4. Genovese, C. R., Lazar, N. A. \& Nichols, T. Thresholding of statistical maps in functional neuroimaging using the false discovery rate. Neuroimage 15, 870-878 (2002).

5. Seeley, W. W. et al. Dissociable intrinsic connectivity networks for salience processing and executive control. J Neurosci 27, 2349-2356 (2007).

6. Del Fabro, L. et al. Functional brain network dysfunctions in subjects at high-risk for psychosis: A meta-analysis of resting-state functional connectivity. Neurosci Biobehav Rev 128, 90-101 (2021).

7. Balaev, V., Orlov, I., Petrushevsky, A. \& Martynova, O. Functional connectivity between salience, default mode and frontoparietal networks in post-stroke depression. J Affect Disord 227, 554-562 (2018).

8. Zhang, $\mathrm{H}$. et al. Aberrant functional connectivity in resting state networks of ADHD patients revealed by independent component analysis. BMC Neurosci 21, 39 (2020).

9. La Corte, V. et al. Cognitive Decline and Reorganization of Functional Connectivity in Healthy Aging: The Pivotal Role of the Salience Network in the Prediction of Age and Cognitive Performances. Front Aging Neurosci 8, 204 (2016).

10. Fang, X. et al. Resting-State Coupling between Core Regions within the Central-Executive and Salience Networks Contributes to Working Memory Performance. Front Behav Neurosci 10, 27 (2016).

11. Murray, L. et al. Sex differences in functional network dynamics observed using coactivation pattern analysis. Cogn Neurosci 1-10 (2021).

\section{Figures}




\section{A}

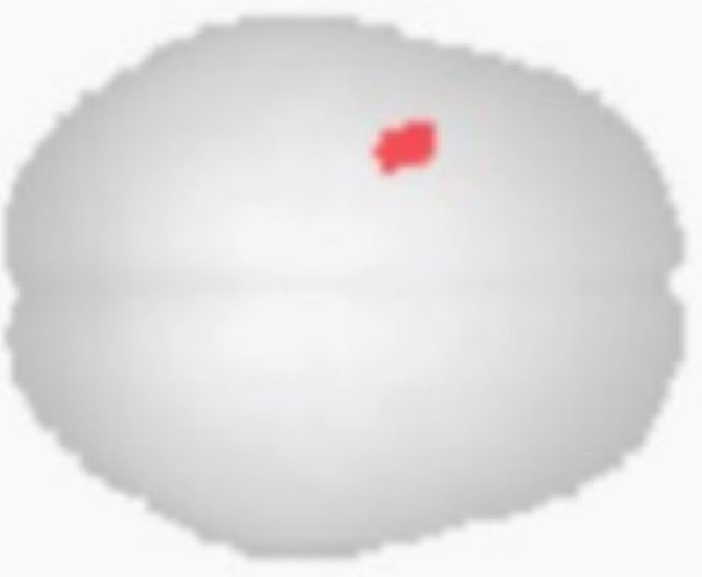

Cluster $(x, y, z)$

sire sixe p-fue sixe p-Foh

sine p-ust

peak p-fut peak p-unc

$-38+02+64$

151 0.017484

อ.012910

c.

๑. 191036

จ. eงece1

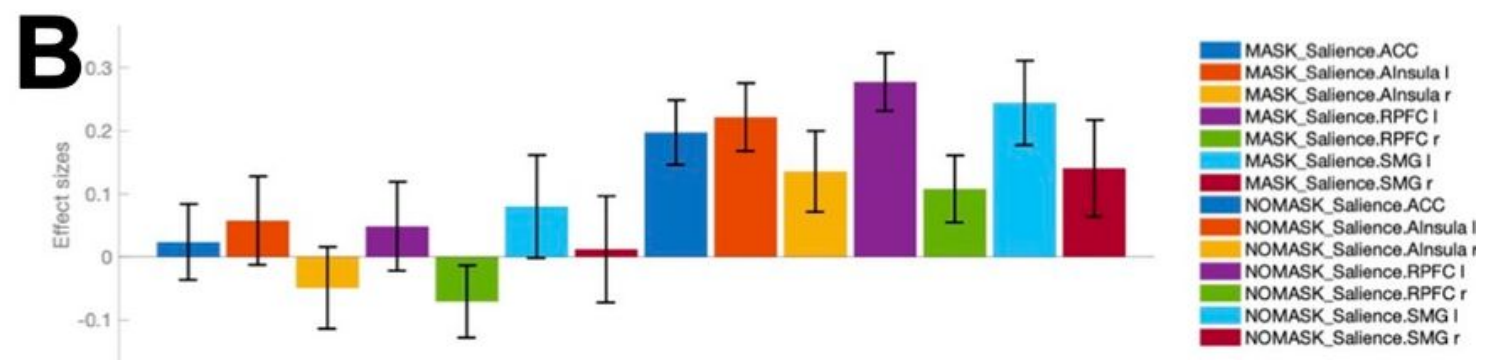

Figure 1

A) The seed-to-voxel analysis with the salience network (SN) as the seed region revealed a significant cluster for wearing a facemask (MASK) versus not wearing a facemask (NO MASK) in the left middle frontal and precentral gyrus. 
B) The detailed analysis of this seed-to-voxel analysis cluster illustrates that the activation effect size of this cluster is lower (arbitrary units) for all seven subregions of the SN when wearing a facemask (MASK left) than for the corresponding regions without wearing a facemask (NOMASK, right). 